\title{
Assessing a retrieval account of the generation and perceptual-interference effects
}

\author{
Neil W. Mulligan and Daniel Peterson \\ University of North Carolina, Chapel Hill, North Carolina
}

\begin{abstract}
A number of memory phenomena are modulated by experimental design, with the effect (e.g., of bizarreness, generation, or perceptual interference) occurring in recall for mixed-list, but not pure-list designs. These effects have other similarities and have been treated in common theoretical frameworks, some focusing on encoding and others on retrieval. The typical paradigm for examining design effects confounds encoding and retrieval contexts, making it difficult to compare these accounts. Using a new paradigm, McDaniel, Dornburg, and Guynn (2005) concluded that retrieval processes contribute to the bizarreness effect. We applied this paradigm to the related perceptual-interference and generation effects. Participants were presented with two pure study lists and later recalled the lists separately (inducing pure retrieval sets) or together (inducing a combined or mixed retrieval set) in a single test. In four experiments, the combined recall condition consistently failed to enhance the size of the generation or perceptual-interference effect. Two additional experiments verified that perceptual interference and generation enhanced recognition memory, as predicted by the standard encoding accounts. The results provide no support for the retrieval account of these two variables but generally are consistent with an encoding locus.
\end{abstract}

A number of variables that generally improve human memory are subject to an important limitation based on experimental design (McDaniel \& Bugg, 2008; Roediger, 2008). For example, consider the generation effect: the finding that self-generated information is remembered better than information perceived passively (Slamecka \& Graf, 1978; see Mulligan \& Lozito, 2004, for a recent review). In a typical study of this effect, participants generate some study words (perhaps from antonyms or anagrams) and read others. When the read and generate trials are intermixed randomly on the study list (a mixed-list design), the generated items produce better recall. However, if the read and generate items are presented in separate study lists, either between or within subjects in blocks (a pure-list design), generation often fails to enhance recall (e.g., Hirshman \& Bjork, 1988; Mulligan, 2001; Mulligan \& Lozito, 2004; Slamecka \& Katsaiti, 1987). The effect of bizarre imagery is likewise influenced by design. In mixed-list designs, bizarre sentences (e.g., "the maid licked the ammonia off the table") produce better recall than do ordinary sentences (e.g., "the maid wiped the ammonia off the table"). However, in a pure-list design, the two sentence types produce equivalent recall (McDaniel \& Einstein, 1986; Worthen, 2006). A third such variable is perceptual interference. In this manipulation, words are presented either in an intact condition, in which the word is read easily, or in a perceptual-interference condition, in which the word is presented very briefly (e.g., $100 \mathrm{msec}$ ) and then backward masked. The perceptual-interference condition often leads to better memory (Hirshman \& Mul- ligan, 1991; Mulligan,1996; Nairne, 1988). Importantly for present purposes, perceptual interference enhances recall in a mixed -list design. but typically not in a pure-list design (Mulligan, 1999, 2002a).

A number of other memory phenomena also are modulated by experimental design (for reviews, see McDaniel \& Bugg, 2008; Schmidt, in press). The effect of orthographic distinctiveness (superior recall for words with unusual spellings) occurs with mixed but not pure lists (Hunt $\&$ Elliott, 1980). Humorous sentences produce better recall than do nonhumorous sentences in mixed lists, but not in pure lists (Schmidt, 1994). Likewise, the enactment effect (superior recall for actions carried out by oneself vs. observed actions) is robust in mixed-list designs but often is eliminated in pure-list designs (e.g., Engelkamp \& Dehn, 2000). Taboo words are recalled better than are neutral words in a mixed list, but not in a pure list (Hadley \& MacKay, 2006). Similarly, Talmi, Luk, McGarry, and Moscovitch (2007) report that emotional pictures produce greater recall than do neutral pictures in mixed but not pure lists. Finally, the word frequency effect in recall is modulated by experimental design, although in a somewhat different way than the other effects. As compared with low-frequency words, high-frequency words produce better recall in pure lists, but often not in mixed lists.

Design effects are important in several ways. First, at an empirical level, they indicate commonality among a diverse set of memory manipulations, a commonality that has been extended to other aspects of memory, such as order memory (e.g., DeLosh \& McDaniel, 1996; En-

N.W. Mulligan, nmulligan@unc.edu 
gelkamp \& Dehn, 2000; Hunt \& McDaniel, 1993; McDaniel \& Bugg, 2008; Mulligan, 1999; Nairne, Riegler, $\&$ Serra, 1991; Schmidt, in press). Second, design effects have been taken as part of the evidence for the relativity of laws of memory (Roediger, 2008). Third, the design limitation has played an important role in theoretical accounts, both in general, unifying accounts (e.g., Hunt \& McDaniel, 1993; McDaniel \& Bugg, 2008; Mulligan \& Lozito, 2004; Schmidt, in press) and in specific accounts of the individual manipulations (e.g., Engelkamp \& Dehn, 2000; Hadley \& MacKay, 2006; Hirshman \& Bjork, 1988; Mulligan, 1999; Nairne et al., 1991; Slamecka \& Katsaiti, 1987; Steffens \& Erdfelder, 1998; Talmi et al., 2007).

Initial demonstrations of design effects prompted artifact accounts. For example, Slamecka and Katsaiti (1987) argued that the generation effect was due to displaced rehearsal, such that generate items were rehearsed preferentially in mixed lists but could not receive such preferential rehearsal in pure lists. Similarly, Begg and Snider (1987) argued that the presence of generate items in a mixed list induced an artificially low encoding goal of mere identification, which especially disadvantages the read items. Similar accounts have been proposed for the bizarreness, word-frequency, and perceptual-interference effects (see Hirshman, Trembath, \& Mulligan, 1994; McDaniel \& Bugg, 2008; Westerman \& Greene, 1997).

Design effects are also central to nonartifact theories, which attempt to unify disparate memory phenomena. The multifactor account and the item-order framework are related accounts that provide an explanation for the effects of bizarreness, generation, perceptual interference, enactment, and word frequency that hinges on the design effects common to them all (Hunt \& McDaniel, 1993; McDaniel \& Bugg, 2008; Mulligan \& Lozito, 2004; Steffens $\&$ Erdfelder, 1998). Both views argue that each of these manipulations contrasts an unusual encoding condition (e.g., generation, perceptual interference, bizarre sentences) with a more usual condition (e.g., reading, intact word presentation, common sentences). The accounts propose that the unusual items attract attention to the processing (and encoding) of individual item characteristics at the expense of encoding interitem relational information (in the case of the item-order account, the particular focus is on the interitem relational information that subserves order memory). In pure lists, the unusual condition would thus produce better item but worse relational (or order) encoding. In mixed lists, however, the disruption to interitem information affects both the unusual and the proximal usual items, producing enhanced item information for the unusual items coupled with equivalent relational (or order) information across conditions. Assuming that both item and interitem relational information contribute to free recall, the enhanced item and disrupted relational (or order) information in the unusual pure lists would counteract one another in recall, generating a reduced or null effect, whereas the enhanced item and equivalent relational (or order) information would produce the typical unusual advantage on recall with mixed lists. ${ }^{1}$ Parenthetically, it should be noted that the full body of research on these effects is more consistent with the multifactor and item-order accounts than with the artifact accounts (McDaniel \& Bugg, 2008; Mulligan \& Lozito, 2004).

The standard accounts of these variables (e.g., bizarreness, generation, perceptual interference, enactment) focus on encoding processes. These accounts argue that varying the experimental design affects the nature of the encoding processes carried out, implying that the later recall test simply reveals this differential encoding. In contrast, other accounts, typically framed in terms of distinctiveness, propose that retrieval processes contribute to the typical (bizarreness, generation, perceptual-interference, word-frequency) effect and to the modulation produced by experimental design (Hunt \& McDaniel, 1993; McDaniel, Dornburg, \& Guynn, 2005; McDaniel \& Geraci, 2006; Schmidt, in press). To be sure, such accounts propose that different study conditions affect encoding, but these accounts also argue that encoding differences interact with the retrieval context to produce observable effects. For example, it has been argued that bizarreness, generation, and perceptual interference all enhance distinctiveness (e.g., Begg, Snider, Foley, \& Goddard, 1989; Gardiner \& Hampton, 1988; Hunt \& McDaniel, 1993; McDaniel, DeLosh, \& Merritt, 2000; Peynircioğlu \& Mungan, 1993). Furthermore, the manifestation of distinctiveness is argued to depend highly on the set of items that are recruited as part of the retrieval set $(\mathrm{McDaniel}$ et al., 2005). We use the term retrieval set as intended by McDaniel et al. (2005; see also Hunt \& McDaniel, 1993) to refer to the set of items (or details) that share a common spatiotemporal context. In an experiment, usually this context is specified (at least implicitly) by the task instructions (e.g., "recall the words from the list you just read"). More generally, it is assumed that all episodic retrieval involves a retrieval set constrained by the (at least implicit) retrieval request. ${ }^{2}$

Retrieval accounts propose that, if all of the items in the retrieval set share the same features, there is no relative distinctiveness and, therefore, no memory advantage. Applied to design effects, this notion proposes that, in the pure-list condition, all items in the retrieval set are of the same (usual or unusual) type and, thus, none is relatively distinct. A lack of relative distinctiveness produces the oft-found null effect in recall. In the mixed-list condition, the retrieval set encompasses an assortment of usual and unusual items, conferring relative distinctiveness onto the unusual condition, which in turn produces the typical effect in recall. This possibility has been raised with several variables that are subject to design effects, such as generation and bizarreness (Begg et al., 1989; Cox \& Wollen, 1981; McDaniel et al., 2000; McDaniel et al., 2005; Schmidt, in press).

Encoding and retrieval accounts propose very different explanations of design effects in memory research. However, as McDaniel et al. (2005) recently pointed out, design effects are ambiguous with respect to the distinction between encoding and retrieval accounts. In the typical paradigm, a participant is presented with a mixed or pure study list, followed by a recall test. McDaniel et al. (2005) argued that the study-list manipulation varies not only the encoding context (as intended), but also the retrieval con- 
text. In the mixed-list condition, the retrieval set consists of items of both types, whereas in the pure-list condition, the retrieval set is homogeneous, consisting of items of a single type. Thus, the pattern of results (the typical effect in the mixed-list condition and its absence or diminution in the pure-list condition) could be due to the study list structure, to the retrieval set, or to both. From the perspective of encoding accounts, this pattern stems from changes in encoding across pure and mixed encoding conditions. From the perspective of retrieval accounts, the operative factor is the retrieval set. When the retrieval set consists of a mixed set of items, the unusual (e.g., bizarre, generate, perceptual-interference) items are relatively distinct and have a retrieval advantage. In contrast, when the retrieval set consists of only one type of item, the unusual items are no longer relatively distinct, and their retrieval advantage is reduced or eliminated.

McDaniel et al. (2005) proposed a new experimental paradigm to decouple the retrieval set from the study list type and used this paradigm to investigate encoding and retrieval accounts of the bizarreness effect. In that experiment, participants were presented with two pure lists of either bizarre or common sentences (counterbalanced for order) separated by a 5 -min distractor task. During the test phase, half of the participants were given a recall test for List 1 and then given a recall test for List 2 (or vice versa). The other half were given a combined recall test in which they were asked to recall any of the sentences from either list. The test manipulation was designed to vary the retrieval set. In the separate recall condition, each test should induce a pure retrieval set, with items of only one type. In the combined condition, the retrieval set was mixed, consisting of items from both lists (and both sentence types). Because encoding conditions were held constant, the encoding accounts argue that the effect of bizarreness should be constant across test conditions. Furthermore, because pure lists were used, the encoding accounts predict little effect of bizarreness in either recall condition. In contrast, the retrieval accounts argue that the combined test condition should induce a mixed retrieval set, rendering the bizarre items relatively distinct (producing a bizarreness effect), whereas the separate test condition should induce pure retrieval sets, no relative distinctiveness, and no bizarreness effect. McDaniel et al.'s (2005) results corresponded to the retrieval account: A significant bizarreness effect was found in the combined condition, but not in the separate condition.

McDaniel et al.'s (2005) results are important for several reasons. First, design effects generally have been interpreted in terms of encoding accounts (McDaniel \& Bugg, 2008; Schmidt, in press). McDaniel et al.'s (2005) results support a retrieval-based alternative. Second, as noted earlier, design effects are important for a number of experimental variables and for theoretical accounts thereof. McDaniel et al.'s (2005) results imply that our understanding of design effects may be incomplete. Third, these results imply that related variables, such as generation, perceptual interference, and enactment, may be amenable to a similar retrieval-based account, because these variables share a number of other empirical regularities (e.g., design effects, their effects on order and relational information; e.g., McDaniel \& Bugg, 2008; Mulligan \& Lozito, 2004).

The present experiments examined the contributions of retrieval processes to the perceptual-interference and generation effects, using the design of McDaniel et al. (2005). In these experiments, participants were presented with two pure lists, one consisting of words in the unusual condition (i.e., the perceptual interference or generate condition) and the other consisting of words presented in the usual (intact or read) condition. As in McDaniel et al. (2005), each study list was followed by $5 \mathrm{~min}$ of distraction to help differentiate the lists at retrieval. Later participants were given separate recall tests or a single, combined recall test. As was detailed earlier, the retrieval account argues that the nature of the retrieval set influences the size of the memory effect. Specifically, separate recall tests should induce pure retrieval sets, whereas a combined recall test is more likely to induce a combined retrieval set, leading to a larger perceptualinterference or generation effect in the latter condition. Accounts focused on encoding predict no difference across recall conditions, because the encoding conditions are held constant. Experiment 1 begins this inquiry with the perceptual-interference manipulation.

\section{EXPERIMENT 1}

\section{Method}

Participants. Thirty-two undergraduates at the University of North Carolina participated in exchange for credit in psychology courses.

Design and Materials. The encoding condition (intact or perceptual interference) was manipulated within subjects, and the recall condition (separate or combined) was manipulated between subjects. Participants were presented with two study lists, one composed entirely of items in the intact condition and the other composed entirely of perceptual-interference items. The order of two lists was counterbalanced across participants. Each list consisted of 16 critical words and 4 additional words that served as primacy (2) and recency (2) buffers. The critical items (as well as the buffer items) were common four- and five-letter words with Kučera-Francis (1967) frequencies of between 100 and 500 . The two lists were presented equally often in the intact and perceptual-interference conditions.

Procedure. Participants were tested individually. Before being given the first study list, participants were told that they would read aloud a series of words presented on a computer screen. They were instructed to try to remember the words for a later (unspecified) memory test. The nature of the study word presentation (i.e., intact or perceptual interference) was also described. In the perceptualinterference condition, each study trial began with a prompt consisting of the words "get ready" and a plus sign "+" centered below, displayed for $500 \mathrm{msec}$. Next, the study word was presented in the same position as the plus sign for $100 \mathrm{msec}$, followed by a row of $\mathrm{Xs}$ in the same position for $2,400 \mathrm{msec}$. In the intact condition, each trial began with the same "get ready" prompt, after which the study word was presented continuously for $2.5 \mathrm{sec}$ (there was no backward mask). The experimenter recorded the participants' responses but provided no feedback.

Following the completion of the first study list, participants were given a 5-min distractor task in which they solved arithmetic problems. The distractor sheet consisted of 70 addition, subtraction, multiplication, and division problems. Participants were instructed to try to solve each problem in their heads without making intermediate calculations on the sheet, writing down only the final answer. The problem set was difficult enough that none of the participants 
finished all of the problems in the $5 \mathrm{~min}$. As in McDaniel et al. (2005), the distractor task was designed to make the lists more easily separated in the later recall period and to clear working memory of local contextual information to further segment the lists. The distractor task was followed by the second study list, which in turn was followed by another 5-min distractor task (a second set of 70 arithmetic problems).

At test, participants were assigned randomly to either the combined or the separate recall condition. In the combined condition, participants were presented with a blank sheet of paper and were asked to write down as many words as they could recall from either study list. The recall test lasted $5 \mathrm{~min}$, and participants were encouraged to use the entire recall period to remember the words. In the separate recall condition, participants were given two recall tests. For half of these participants, the first test referred to the first study list and the second referred to the second study list; the order of the recall tests was reversed for the other half. For each recall test, participants were first asked to think back to one specific list of words (i.e., either the first or the second list). Participants were reminded of the form in which they had seen the words (e.g., "Those were the words presented only briefly, then covered with a row of Xs"). Participants were instructed to write down all the words they could remember from that list. After $2.5 \mathrm{~min}$, participants were asked to stop and then were given instructions for the second recall test. During this portion, participants were asked to think back to the other list of words (the list not recalled previously), reminded of the manner of presentation for that list, and were given $2.5 \mathrm{~min}$ to write down as many of those words as possible.

\section{Results and Discussion}

At study, participants correctly identified all of the words in the intact condition and $99.6 \%$ in the perceptualinterference condition. Table 1 presents performance on the recall phase. The separate recall condition was scored in two ways. The stringent recall score required the item to be recalled on the correct test, and the lenient score considered an item correctly recalled if it was reported on either test. Both scores yielded the same pattern of results in this and later experiments (with one exception noted in Experiment 3); only the stringent recall scores will be reported here. The proportions recalled were submitted to a $2 \times 2$ ANOVA, using encoding condition and recall test as factors (the alpha level was set to .05 for this and subsequent analyses). The analysis revealed no significant effects $(p s>.25)$.

First, consider the results of the separate recall test. According to the logic of the paradigm developed by McDaniel et al. (2005), this condition should be similar to a traditional pure list design in which a test follows a single list and the retrieval set is unmixed. In pure-list designs, perceptual interference typically fails to enhance

Table 1

Experiment 1: Mean Proportions of Items Recalled, With Standard Errors, As a Function of Encoding and Recall Conditions

\begin{tabular}{cccccc}
\hline & \multicolumn{3}{c}{ Encoding Condition } \\
\cline { 2 - 6 } & $\begin{array}{c}\text { Perceptual } \\
\text { Interference }\end{array}$ & \multicolumn{2}{c}{ Intact } \\
\cline { 2 - 6 } Recall Condition & $M$ & $S E$ & & $M$ & $S E$ \\
\hline Separate & .16 & .03 & .16 & .03 \\
Combined & .21 & .04 & .20 & .03 \\
\hline
\end{tabular}

recall (Mulligan, 1999). Consistent with this result, the separate recall condition produced no effect of perceptual interference. Of course, as McDaniel et al. (2005) pointed out, the results of this condition are ambiguous with regard to the distinction between encoding and retrieval accounts, necessitating the use of the combined recall condition. Here, we see no evidence for the emergence of the perceptual-interference effect and thus no evidence for the retrieval account.

A potential concern is that participants in the combined condition failed to form a combined retrieval set but, rather, recalled the items separately from Lists 1 and 2, which would have rendered the combined condition similar to the separate condition. Such a strategy would result in recall clustering by list. To assess this, we used the adjusted-ratio-of-clustering (ARC) score (Roenker, Thompson, \& Brown, 1971), a common measure of clustering that has a value of 0 for chance-level clustering, positive values for above-chance clustering, and a value of 1 for perfect clustering. The mean ARC score was .03 , not significantly different from $0(|t|<1)$, indicating no evidence for a list-based retrieval strategy in the combined condition.

\section{EXPERIMENT 2}

Although Experiment 1 used the same perceptualinterference manipulation that had been found to enhance memory in earlier studies (see Mulligan \& Lozito, 2004, for a review), the results may raise the concern that the manipulation was simply ineffective with the present materials or list structures. If so, the results of Experiment 1 would be uninformative with respect to encoding or retrieval accounts. The standard encoding views argue that perceptual interference, like generation, enhances the encoding of item-specific information (Mulligan, 1999; Mulligan \& Lozito, 2004). Consequently, perceptual interference is predicted to enhance recognition memory even in a pure-list design because recognition relies heavily on item information and little on relational or order information. Experiment 2 replaced the recall phase of Experiment 1 with a recognition test to make sure that the perceptual-interference manipulation was effective under the present encoding circumstances.

\section{Method}

Participants. Sixteen undergraduates at the University of North Carolina participated in exchange for credit in psychology courses.

Design and Materials. The design and materials were identical to those of Experiment 1, with the following changes. Instead of a recall test, participants completed a test of recognition memory that consisted of the 32 critical words from both study lists and 32 new words chosen to be similar in frequency and length to the critical words. ${ }^{3}$ The test list was ordered randomly, with the constraint that no type of item (old or new) was repeated more than twice in a row.

Procedure. The encoding procedures (study list and distractor tasks) were identical to those in Experiment 1. At test, participants were presented with an old/new recognition test. They were told that they would be presented with a series of words, some that had been presented previously during the study portion (old) and some that were new. Participants were instructed to indicate their decision by 
pressing the "o" key for old or the "n" key for new. Each test word was presented on the computer screen until the participant responded.

\section{Results and Discussion}

At study, participants correctly identified all of the words in both the intact and perceptual-interference conditions. On the recognition test, the mean proportions of hits were .87 and .78 in the perceptual-interference and intact conditions, respectively (the false alarm rate was .25). Perceptual interference produced significantly greater recognition accuracy than the intact condition did, regardless of whether it was measured by corrected hit rate $[t(15)=2.30]$ or by $d^{\prime}$ (mean $d^{\prime}$ s of 2.15 and 1.69, respectively) $[t(15)=2.34]$.

Perceptual interference enhanced recognition accuracy relative to the intact condition. This contrasts with the results of Experiment 1, in which the present pure-list design failed to affect recall. This pattern of results is consistent with the standard encoding view, arguing that perceptual interference enhances item-specific encoding even though it may harm relational and order encoding (Mulligan \& Lozito, 2004). For pure-list designs, this analysis argues that recognition should exhibit a perceptual-interference effect because recognition is sensitive primarily to item information and is relatively insensitive to relational/ order information. Furthermore, the present results replicate those in earlier research using more standard implementations of the pure-list design (Mulligan, 1999, 2000). Most importantly, the present results indicate that the perceptual-interference manipulation used in Experiment 1 is an effective manipulation, given the present materials, list structure, and so forth. This permits greater confidence in interpreting the results of Experiment 1 as inconsistent with the retrieval account.

\section{EXPERIMENT 3}

The generation effect is another of the variables moderated by experimental design. Like the effects of bizarreness, perceptual interference, word frequency, and enactment, the generation effect is encompassed by the multifactor and item-order accounts (McDaniel \& Bugg, 2008; Mulligan \& Lozito, 2004; Steffens \& Erdfelder, 1998), both of which are encoding accounts. Likewise, the generation effect has been attributed to distinctiveness in accounts that argue for the contribution of retrieval processes (e.g., Begg et al., 1989; Hunt \& McDaniel, 1993; McDaniel \& Geraci, 2006; Peynircioğlu \& Mungan, 1993). Experiment 3 applied the same design as that in Experiment 1 (and McDaniel et al., 2005, Experiment 1) to the generation manipulation. In particular, participants generated the words of one study list (by transposing letters; see, e.g., Kinoshita, 1989; Mulligan, 2002b; Nairne \& Widner, 1988) and read the words of the other study list. The retrieval account predicts that the combined retrieval set will enhance the relative distinctiveness of the generated items and will produce a generation effect larger than that in the separate condition. Encoding views argue that the retrieval manipulation will leave the generation effect unchanged.

\section{Method}

Participants. Thirty-two undergraduates at the University of North Carolina participated in exchange for credit in psychology courses.

Design and Materials. The encoding condition (read or generate) was manipulated within subjects and recall condition (separate or combined) was manipulated between subjects. The study words were the same as those of Experiment 1. Read items were presented in their normal form, and generate items were presented with the first two letters transposed (e.g., rbain for brain).

Procedure. The encoding procedures (including study list and distractor tasks) were identical to those in Experiment 1, except for the following modifications. Before the generate list, participants were told that each item would be presented with the first two letters reversed and that they should transpose the letters to generate the word, writing it on their answer sheet. Participants were also told to try to remember the words for the later (unspecified) memory test. The generate items were presented individually on the computer screen for $5 \mathrm{sec}$, allowing enough time to generate and write down the words. In the read condition, participants were instructed to write down each word and to try to remember it for the memory test. These items were presented individually every $5 \mathrm{sec}$ in their normal form. The recall phase was identical to that in Experiment 1.

\section{Results and Discussion}

At study, participants correctly generated $99.7 \%$ of the words (and correctly copied all of the words in the read condition). Table 2 presents performance in the recall phase. The proportions recalled were submitted to a 2 (encoding condition) $\times 2$ (recall condition) ANOVA. The analysis revealed a significant effect of encoding condition $\left[F(1,30)=10.62, M S_{\mathrm{e}}=.020\right]$, indicating that generation enhanced recall. The effect of test condition and its interaction with encoding condition were not significant $(F \mathrm{~s}<1) .{ }^{4}$ As in Experiment 1, the mean ARC score $(-.01)$ in the combined condition was not significantly different than zero $(|t|<1)$, indicating no evidence for a list-based retrieval strategy.

The central result is that the generation effect is not influenced by recall condition. The retrieval account argues that the effect should be enhanced in the combined condition, as compared with the separate test condition, whereas encoding accounts predict no such difference. As in Experiment 1, the results favor an encoding view. It should be noted that, even though there was no interaction between the encoding and test conditions, there was a main effect of generation. Given that the present paradigm is a type of pure-list design, this result might seem surprising. We have two points on this issue. First, an effect of generation in a pure-list design is certainly not unprecedented (see Bertsch, Pesta, Wiscott, \& McDaniel, 2007, for a review). Second, from the perspective of the

Table 2

Experiment 3: Mean Proportions of Items Recalled, With Standard Errors, As a Function of Encoding and Recall Conditions

\begin{tabular}{cccccc}
\hline & \multicolumn{3}{c}{ Encoding Condition } \\
\cline { 2 - 3 } \cline { 5 - 5 } Renerate & & \multicolumn{2}{c}{ Read } \\
\cline { 2 - 3 } \cline { 5 - 6 } Recall Condition & $M$ & $S E$ & & $M$ & $S E$ \\
\hline Separate & .31 & .03 & & .20 & .03 \\
Combined & .29 & .04 & & .16 & .04 \\
\hline
\end{tabular}


encoding accounts, the expectation is not that there will be a complete absence of an effect with pure lists but, rather, that the effect will be reduced in pure-list, relative to mixed-list, designs (which is generally the case; Bertsch et al., 2007). The encoding views do not predict that the offsetting effect of item and order/relational encoding will always be perfect (i.e., producing no effect in recall). Consequently, an absolute absence of a generation effect is not a prerequisite for the present results to be viewed as inconsistent with the retrieval account.

Although the main effect of generation in Experiment 3 is not unprecedented, it deserves further scrutiny. From the perspective of the retrieval account, a plausible concern is that the two study lists were difficult to differentiate at retrieval. If so, the retrieval sets in the separate condition were mixed functionally due to the participants' inability to differentiate the lists. Under this retrieval account, both retrieval conditions may have been based on combined retrieval sets, and both would be expected to exhibit an effect of generation. This analysis also implies that participants would make frequent list confusion errors in the separate condition (recalling List 1 items on the List 2 test, and vice versa).

This retrieval-based analysis comports with the main result of Experiment 3, but there are reasons to question it. First, Experiment 1 failed to show an effect of perceptual interference when the same design and materials were used. If participants in the separate condition of the present experiment could not differentiate the lists at retrieval (and this fact accounts for the observed generation effect), there is no reason that this would have been any easier in Experiment 1 . Yet no main effect of perceptual interference was found in that experiment. Second, it should be noted that the experimental design used in both of these experiments was the same as that used by McDaniel et al. (2005), in which list differentiation was not considered to be a problem. Third, the number of list confusions in Experiment 3 was not particularly high, averaging less than one list confusion error on each test in the separate condition $(M=$ 0.94). Finally, given even a modest, but imperfect, ability to differentiate the lists, the retrieval account implies that the separate condition should still produce less retrieval distinctiveness (and a smaller generation effect) than the combined condition. Again, this is not measurably the case.

Regardless, we decided that additional experimentation was required to rule out this retrieval-based account of the results of Experiment 3. To do so, we modified the materials of Experiment 3 to ensure greater list differentiation. In Experiment 4, all of the words in one study list came from one taxonomic category whereas all the words in the other list came from a different taxonomic category. This should enhance the ability of participants in the separate condition to differentiate clearly the lists and to form purer retrieval sets.

\section{EXPERIMENT 4}

\section{Method}

Participants. Twenty-four undergraduates at the University of North Carolina participated in exchange for credit in psychology courses.
Design, Materials, and Procedure. Experiment 4 was identical to Experiment 3, except for the following modifications. Sixteen critical words were chosen from each of two categories (fruit and animal) in the Battig and Montague (1969) norms. The mean category rank for the critical items was 21.5. To discourage guessing on the recall tests, very common examples (with category ranks less than 10) were not selected. An additional four items from each category were chosen to serve as primacy and recency buffers for the two study lists. The study lists were homogenous with respect to encoding condition and category. Both the study and test instructions mentioned the categorical structure of the study lists. Specifically, prior to each study list, the encoding task was described (either read or generate), and it was mentioned that that all of the items were from the same category (either fruit or animal). In the separate recall condition, participants were instructed to recall only one list at a time and were reminded of the list's encoding condition and category (e.g., "Those were the animal names presented with the reversed letters"). Likewise, the combined recall instructions mentioned the encoding condition and category of each list but emphasized that any word presented in either list was to be recalled.

\section{Results and Discussion}

At study, participants correctly generated and copied down all of the words. The proportions recalled (Table 3 ) were submitted to a 2 (encoding condition) $\times 2$ (recall condition) ANOVA, which revealed no significant main effects and no interaction $(F \mathrm{~s}<1)$. It should be noted that recall was quite a bit better in the present experiment (averaging .56) than in Experiments 1 and 3 (which averaged .21), reflecting the typical finding that organized lists produce better recall than do random lists (e.g., Hunt \& McDaniel, 1993).

As in Experiments 1-3, the critical result in this experiment is that the generation effect is not influenced by the test condition. In this experiment, steps had been taken to ensure that the study lists were differentiated more easily than in Experiment 3. In that experiment, there had been a potential concern that in the separate condition, the lists could not be adequately differentiated at retrieval. Using different categories for the two lists should make this easier, yet the same critical result was obtained: The effect (in this case, null effect) of generation was unchanged by the recall condition. This is inconsistent with the retrieval-based account of Experiment 3 and further indicates that the combined recall condition does not lead to an enhanced generation effect. ${ }^{5}$

The lack of a main effect of generation requires comment. Although pure-list manipulations often produce a null effect of generation, researchers have sometimes found that pure-list manipulations coupled with categorized target items (as opposed to unrelated target items)

Table 3

Experiment 4: Mean Proportions of Items Recalled, With Standard Errors, As a Function of Encoding and Recall Conditions

\begin{tabular}{|c|c|c|c|c|}
\hline \multirow[b]{3}{*}{ Recall Condition } & \multicolumn{4}{|c|}{ Encoding Condition } \\
\hline & \multicolumn{2}{|c|}{ Generate } & \multicolumn{2}{|c|}{ Read } \\
\hline & $M$ & $S E$ & $M$ & $S E$ \\
\hline Separate & .57 & .05 & .56 & .05 \\
\hline Combined & .54 & .04 & .56 & .05 \\
\hline
\end{tabular}


can produce a significant generation effect in recall (e.g., deWinstanley, Bjork, \& Bjork, 1996; McDaniel, Riegler, $\&$ Waddill, 1990). This result is part of the evidence in favor of the multifactor account (Mulligan \& Lozito, 2004). Critical to the production of this result is that the category relations among the target items be useful (and used) in generating the target item. The present results appear to conflict with these findings, and there are at least two possibilities why. First, in the present experiment, the target items could be generated easily (by reversing the first two letters) without reference to the shared category. If participants do not make use of the shared relational information during the act of generation, the multifactor model predicts no generation effect, and none has been found empirically (e.g., Burns, 1990; McDaniel et al., 1990; Steffens \& Erdfelder, 1998). That is, the mere presence of categorized materials does not produce a purelist generation effect (Burns, 1990; Steffens \& Erdfelder, 1998). Second, in the earlier research, study lists consisted of a smaller number of items (e.g., six) from each of several categories, whereas in the present experiment, each study list consisted of a larger number of items from a single category. The pure-list generation effect may be modulated by the number of examples associated to the category cue (e.g., as in a cue-overload effect).

Although the results of Experiments 1, 3, and 4 provide little support for the retrieval account, before rejecting this hypothesis we decided to err on the side of caution by entertaining one more retrieval-based account. The potential, retrieval-based concern about Experiment 3 was that list differentiation may have been too difficult. The opposite retrieval-based concern might be raised about Experiment 4: Perhaps the attempt to make list differentiation easier succeeded too well and caused participants in the combined condition to spontaneously organize their retrieval attempts around list/category membership. For Experiment 3 , the concern was that poor list differentiation rendered the separate condition functionally equivalent to the combined condition. The complementary concern regarding Experiment 4 is that too-easy list differentiation rendered the combined condition functionally equivalent to the separate condition.

Although not definitive, there is some evidence for this possibility. Of 12 participants in the combined condition, 2 explicitly grouped their recall by list/category on the answer sheet (whereas the rest recorded their answers in the order recalled). Of course, this is not an unambiguous indication of a list-based retrieval strategy; these participants may have grouped their answers upon recording. The ARC scores for the 10 participants who did not explicitly group their answers demonstrated a trend toward above-chance clustering [mean ARC $=.24 ; t(9)=1.94, p=.08$ ] .

The possibility of a list-based retrieval strategy in the combined condition prompted Experiment 5. Under the retrieval analysis, Experiment 3 may have made list differentiation too difficult, and Experiment 4 may have made it too easy. Experiment 5 sought to split the difference by introducing a stronger basis for list differentiation than that in Experiment 3 but a basis more subtle than that used in Experiment 4 to reduce the likelihood of a "separate" retrieval strategy in the combined condition. Experiment 5 used the same design as that in Experiments 3 and 4 but used one study list of verbs and another of nouns, an aspect of organization that is less salient than taxonomic category membership, but one that could still be used at retrieval to help differentiate the lists.

\section{EXPERIMENT 5}

\section{Method}

Participants. Thirty-two undergraduates at the University of North Carolina participated in exchange for credit in psychology courses.

Design, Materials, and Procedure. Experiment 5 was identical to Experiments 3 and 4, except for the following modifications. The critical items were a set of 16 verbs and 16 nouns. The critical items were common words from four to seven letters in length with an average Kučera-Francis (1967) frequency of 67. An additional 4 verbs and nouns were selected to serve as primacy and recency buffers for their respective study lists. The study lists were homogenous with respect to encoding condition and verb-noun status. During the study portion, no mention was made of the verb-noun structure of the lists. In the separate recall condition, participants were informed that the list to be recalled consisted of all nouns or all verbs. In the combined recall condition, the participants were not so informed (again, these participants were instructed simply to recall all of the words from both lists). The difference in instructions was designed to ensure that the separate group could capitalize on this subtle basis for list differentiation and that the combined group would be less likely to spontaneously recall by list.

\section{Results and Discussion}

At study, participants correctly generated $99.2 \%$ of the words (and correctly copied all of the words in the read condition). The proportions recalled (Table 4) were submitted to a 2 (encoding condition) $\times 2$ (recall test) ANOVA that revealed no significant main effects and no interaction $\left(F_{\mathrm{S}}<1.6, p \mathrm{~s}>.20\right)$. Clustering by list in the combined recall test was not significantly above chance (mean ARC score of .11; $t=1.22, p>.20$ ). In the separate condition, list confusion errors were extremely few (a single participant made two list confusion errors). The combination of low list confusion errors in the separate condition and little clustering by list in the combined condition indicates that the lists were differentiable in the separate conditions but that the differentiation was subtle enough that it was not spontaneously used as the basis of retrieval in the combined condition. The primary results persist in supporting encoding views, rather than the retrieval view. There is no evidence that the effect of encoding condition was influenced by the retrieval conditions in

Table 4

Experiment 5: Mean Proportions of Items Recalled, With Standard Errors, As a Function of Encoding and Recall Conditions

\begin{tabular}{cccccc}
\hline & \multicolumn{4}{c}{ Encoding Condition } \\
\cline { 2 - 3 } \cline { 5 - 5 } \cline { 5 - 5 } Renerate & & \multicolumn{2}{c}{ Read } \\
\cline { 2 - 5 } \cline { 5 - 6 } & $M$ & $S E$ & & $M$ & $S E$ \\
\hline Separate & .30 & .03 & .28 & .05 \\
Combined & .27 & .04 & .24 & .04 \\
\hline
\end{tabular}


any of the recall experiments (Experiments 1, 3, 4, or 5), regardless of the salience or subtlety of the characteristics that differentiate the study lists.

\section{EXPERIMENT 6}

Experiments 4 and 5 did not exhibit any generation effect, which may raise concerns about the effectiveness of this manipulation in the present case (even though this generation manipulation did affect recall in Experiment 3). As with the perceptual-interference manipulation, the typical encoding view argues that generation enhances item information (Mulligan \& Lozito, 2004), implying an effect on recognition even with pure lists. The present experiment used the study materials and procedures of Experiment 5, followed by a recognition test to verify the manipulation's effectiveness.

\section{Method}

Participants. Thirty-two undergraduates at the University of North Carolina participated in exchange for credit in psychology courses.

Design and Materials. The design and materials were identical to those of Experiment 5, with the following changes. Instead of a recall test, participants completed a test of recognition memory. The recognition test consisted of the 32 critical words from both study lists and 32 new words (half verbs and half nouns) chosen to be similar in frequency and length to the critical words (see note 3 ). The list was ordered randomly, with the constraint that no type of item (old or new) was repeated more than twice in a row.

Procedure. The encoding procedures (including study list and distractor tasks) were identical to those in Experiment 5. The recognition test was identical to that in Experiment 2. For the recognition test, no mention was made of the verb-noun structure of the study lists.

\section{Results and Discussion}

At study, participants correctly identified all of the words in both the read and generation conditions. On the recognition test, the mean proportions of hits were .89 and .75 in the generation and intact conditions, respectively (the false alarm rate was .19). Generation produced significantly greater recognition accuracy than did the read condition whether measured by corrected hit rate $[t(31)=$ 2.79 ] or by $d^{\prime}$ (mean $d^{\prime}$ s of 2.44 and 1.98 , respectively) $[t(31)=2.23]$.

In contrast to its effects on recall in Experiment 5, the present generation manipulation enhanced recognition memory. This pattern of results is consistent with the standard encoding view arguing that generation enhances item-specific encoding and, consequently, enhances recognition even in pure-list designs. Furthermore, the present results replicate those in earlier research showing the standard generation effect in the typical pure-list design in which a single, pure study list is directly followed by a recognition test (see Mulligan \& Lozito, 2004, for a review). Most importantly, the present results indicate that the generation manipulation used in Experiment 5 is an effective manipulation, given the present materials, list structure, and so forth. As with the results of Experiment 2 , this permits greater confidence in interpreting the results of the recall experiments as inconsistent with the retrieval account.

\section{ANCILLARY ANALYSES OF THE RECALL DATA}

A number of ancillary analyses can best be addressed by combining data across the four recall experiments (Experiments 1, 3, 4, and 5). First, the retrieval account predicts an interaction between encoding and test condition, which we repeatedly failed to find. To provide a more powerful analysis, the data from all four of the recall experiments were pooled. This analysis exhibited no hint of an interaction between encoding and recall conditions $(F<1)$, despite the large sample $(N=120)$. This is unlikely to be an issue of low power, since the average encoding effect was slightly (but of course, nonsignificantly) greater in the separate than in the combined condition. If low power was masking an effect, the trend should have been in the opposite direction. In addition, the pooled analysis has more participants (and thus more power) than did the original study by McDaniel et al. (2005, Experiment 1), which showed results consistent with the retrieval account. Given the size of the effect $(f=.48)$ reported by McDaniel et al. (2005, Experiment 1), the presented combined analysis had a power exceeding .99 .

A second potential issue is that some participants in the combined condition may have grouped their recall by study list. Although the clustering measure was not significant in Experiments 1, 3, and 5 (and was only marginally significant in Experiment 4), some participants in each experiment exhibited high values of clustering. According to the retrieval account, such participants are similar to those in the separate condition and should be less likely to show an encoding effect, whereas those exhibiting little clustering should be more likely to show an encoding effect (e.g., Talmi et al., 2007). Of the 60 participants in the combined condition, ARC scores were computable for 55 (ARC could not be computed if a participant failed to recall items for one of the lists or, as in Experiment 4, if the participant overtly grouped responses by lists). The size of the encoding effect was not different for high $(\mathrm{ARC} \geq 0$, $n=31$ ) versus low (ARC $<0, n=24)$ clusterers $(F<1)$. Thus, there is no evidence that the amount of list clustering dictated the present pattern of results.

Third, the main results are unchanged when only the first recall test in the separate condition is considered. In this analysis, the encoding variable is rendered a betweensubjects manipulation for the separate test condition (whereas it remains within subjects in the combined condition), requiring the use of Erlebacher's (1977) method. The interaction between encoding and test conditions was nonsignificant for the pooled data $(F<1)$, as it was when each recall experiment was analyzed individually $(F \mathrm{~s}<1.3)$. This analysis eliminates any potential interference that the first test may have had on the second, and yet the primary result is unchanged: The size of the encoding effect was not affected by retrieval condition.

Researchers investigating the item-order account typically use the input-output (IO) correspondence score to assess order information in free recall (DeLosh \& McDaniel, 1996; McDaniel et al., 2005; Nairne et al., 1991). This measure assesses the extent to which recall output corre- 
sponds to serial input position, with a chance-level value of .5. In the present case, this measure is likely to be suggestive rather than definitive, since IO scores tend to be near chance level for lists of 16 items or more, indicative of little reliance on serial-order information (Mulligan \& Lozito, 2007). In the combined condition, the two study lists were treated as a single list for purposes of computing IO scores. In the separate condition, IO scores were computed for each test, along with an average of the two, to facilitate comparison with the combined condition. To compute IO scores, at least two items must be recalled, preventing computation for some of the recall protocols in the separate condition. In general, the results of the IO scores are as expected. First, average IO scores were relatively low but significantly greater than chance [with an overall average of .56; $t(116)=3.23$ ]. Second, IO scores were significantly above chance in the separate condition [averaged over tests, $M=.58 ; t(56)=2.88]$ and only marginally significant in the combined condition $[M=.54 ; t(59)=1.71$. $p<.10]$. Given that the functional study list was longer in the combined condition, this pattern is consistent with the finding that longer lists exhibit less IO correspondence (Mulligan \& Lozito, 2007). Third, IO scores in the read/ intact condition exceeded chance $[M=.60 ; t(44)=2.96]$, whereas those in the generate/perceptual-interference conditions did not $[M=.54 ; t(54)=1.12]$. This is consistent with the item-order account, which argues for deficient order memory in the generate/perceptual-interference condition but is merely suggestive, since the direct comparison between these conditions was not significant $(t=1.41, p=$ .16). Despite this limitation in the data, the difference was consistent across experiments; within each of the four experiments, the average IO score was numerically higher in the read/intact condition than in the generate/perceptualinterference condition. This pattern is largely consistent with the typical finding that order information is greater in the read/intact condition than in the generate/perceptualinterference condition, although the pattern is more pronounced with shorter lists (e.g., Mulligan, 1999; Nairne et al., 1991).

\section{GENERAL DISCUSSION}

A number of memory effects (e.g., generation, perceptual identification, word frequency, bizarreness, enactment) are modulated by experimental design, raising the potential of important commonalities. These variables have been subjected to common theoretical analyses, some focusing on encoding processes (e.g., DeLosh \& McDaniel, 1996; Engelkamp \& Dehn, 2000; McDaniel \& Bugg, 2008; Mulligan \& Lozito, 2004; Nairne et al., 1991; Steffens \& Erdfelder, 1998) and others focusing on retrieval (e.g., Hunt \& McDaniel, 1993; McDaniel et al., 2000; McDaniel et al., 2005; see Schmidt, in press, for a discussion). Both types of theories provide an account of design effects, but as McDaniel et al. (2005) recently pointed out, the usual experimental paradigm for studying design effects confounds encoding and retrieval context. Using a new paradigm to analyze the bizarreness effect, McDaniel et al. (2005) adduced support for the retrieval account.
The goal of the present experiments was to determine whether this retrieval-based analysis applied to perceptual interference and generation. This account proposes that retrieval contributes to both of these effects (e.g., via relative distinctiveness), and thus both effects should be enhanced by a combined, rather than pure-retrieval set. Accounts focusing on encoding (e.g., item-order and multifactor accounts) imply that the retrieval condition should not influence generation or perceptual interference, because the encoding conditions were held constant. Using the paradigm developed by McDaniel et al. (2005), we contradicted the retrieval account consistently. In Experiment 1 , the effect of perceptual interference was uninfluenced by retrieval condition. Experiments 3-5 showed that the effect of generation was likewise uninfluenced. Experiments 2 and 6 demonstrated that the present manipulations of generation and perceptual interference enhanced recognition memory, obviating the concern that these manipulations were simply ineffective under the present circumstances.

It should be noted that throughout these experiments, efforts were made to assess adequately the retrieval account. First, the experiments made use of the paradigm developed by McDaniel et al. (2005), which was designed to assess the retrieval account and is capable of producing results fully supportive of that account. Second, when the initial experiments (Experiments 1 and 3) yielded results unfavorable to the retrieval account, we assessed supplementary hypotheses needed to make the results conform to the retrieval account. Specifically, we entertained the notion that the original lists were not discriminable enough to provide a fair test of the retrieval hypothesis and set about making the lists more differentiable (Experiment 4). When that experiment also yielded results inconsistent with the retrieval hypothesis, we entertained the notion that the study lists were now too easily discriminated and assessed that notion by instituting list materials that more subtly differentiated the lists (Experiment 5). After having given the retrieval account these additional benefits of the doubt, neither experiment yielded results consistent with the retrieval account.

In addition, the combined analysis of Experiments $1,3,4$, and 5 demonstrated that even with a highpowered analysis, there was no hint of an interaction between encoding and retrieval conditions. The average size of the encoding effect was very nearly equal across the retrieval conditions and in fact was slightly (and, of course, nonsignificantly) greater in the separate condition. Thus, the experiments provide no evidence that retrieval processes drive design effects in the case of perceptual interference or generation (Healy, Shea, Kole, \& Cunningham, 2008 , come to a similar conclusion in research on serial position effects).

The present experiments were designed to test the retrieval account, rather than provide a fine-grained analysis of which encoding account best fits the data. Regardless, it is worth considering the results in terms of the encoding accounts described in the introduction. The artifact accounts (e.g., displaced rehearsal) are not supported, because these views predict no differential processing across 
encoding conditions in pure-list designs, and yet both perceptual interference and generation enhanced recognition (see McDaniel \& Bugg, 2008; Mulligan \& Lozito, 2004, for further evidence against these accounts). The itemorder account argues that both generation and perceptual interference enhance item processing and disrupt order processing and that order information contributes to free recall. The multifactor account argues for a similar tradeoff in item and relational processing. Both accounts are consistent with the findings in general outline. First, in a pure-list design, both the generation and the perceptualinterference effects are found for recognition memory, a test primarily sensitive to item encoding, and are more labile for free recall, a test sensitive to both item and relational processing. Second, being encoding views, both accounts imply that effects in recall should not be moderated by separate versus combined test conditions. However, both accounts face challenges in some of the details of the results. In the case of the item-order account, the IO scores provide mixed support. Consistent with this account, the generation and perceptual-interference conditions generally produced less reliance on order information than did the read and intact conditions. However, the IO scores were not much above chance, and key comparisons were not significantly different. In sum, there is little indication that order information was a key determinant of recall (not unexpected, given the length of the study lists used; Mulligan \& Lozito, 2007).

The multifactor account is the most generally successful account of generation and perceptual-interference effects (Mulligan \& Lozito, 2004) and may be a better candidate for a complete account of the present results. Even here, however, there are questions. Potentially problematic is the lack of a main effect of generation in Experiment 4 , in which categorized materials were used. As was discussed in that experiment, categorized materials often (but not always) produce a generation effect in recall even in a pure-list design. The multifactor account might claim that category information was not used in the act of generation in this experiment, a plausible claim given the ease of the generation task. However, there is no independent evidence for this within the experiment. A more complete assessment of the multifactor account as it applies to the present paradigm requires more comprehensive measurement of item and relational information, using, for example, the gain-loss analysis, measures of subjective organization, or measures of category clustering within lists. All of these techniques have been used to assess the multifactor account in related experimental paradigms and, it should be noted, generally have supported this view (e.g., Hunt \& McDaniel, 1993; Mulligan \& Lozito, 2004; Steffens \& Erdfelder, 1998).

The present results contrast with the results of McDaniel et al. (2005) in which the bizarreness effect was absent in the separate condition but emerged in the combined condition. Although recent theorizing has emphasized the similarities between bizarreness and a variety of other variables (such as generation, perceptual interference, enactment, and word frequency; McDaniel \& Bugg, 2008), there are of course differences among these vari- ables as well. For example, even in mixed-list designs, bizarre items often fail to enhance recognition memory (e.g., McDaniel \& Einstein, 1986; see Worthen, 2006, for a review), whereas generation and perceptual interference consistently enhance recognition in mixed- and pure-list designs (Mulligan \& Lozito, 2004). In general, the effects of bizarreness are demonstrated more consistently in recall than in recognition, whereas the effects of perceptual interference and generation are demonstrated more readily in recognition than in recall (Hirshman \& Mulligan, 1991; Mulligan \& Lozito, 2004; Nairne, 1988). Another example is that the use of categorized materials eliminates the bizarreness effect in a mixed-list design because, as has been argued by McDaniel et al. (2000), the category structure provides an alternative retrieval strategy to the typical distinctiveness-based recall strategy. The effects of generation and perceptual interference are not eliminated by the use of categorized materials in a mixed-list design (e.g., Mulligan, 1999, 2001; Mulligan \& Lozito, 2004; Nairne et al., 1991). This empirical difference may be another indicator that the bizarreness effect is more malleable in the face of retrieval manipulations than are the generation and perceptual-interference effects.

The effects of the present retrieval manipulation appear to be another difference between the bizarreness effect and the generation and perceptual-interference effects. On the basis of the logic of McDaniel et al. (2005), this implies that retrieval processes play a greater role in the bizarreness effect than in the generation or perceptual-interference effects or that, at the very least, retrieval processes are less able to reverse the usual design effects found with the latter variables. At this point, we can only speculate on the reasons for this difference. One salient difference between research on bizarreness and research on generation and perceptual interference is the nature of the "items" used. In the former, sentences or phrases constitute the items, whereas in the latter, individual words are typically the items. It is possible that a distinctiveness-based retrieval strategy is a more usual or effective strategy with more complex items, producing a greater reliance on retrieval processes for bizarreness than for the other two variables. Second, there is evidence that it is easier to form interitem associations with random lists of simple items (e.g., individual words), as opposed to more complex items (e.g., sentences; cf. Engelkamp, Jahn, \& Seiler, 2003; Mulligan \& Lozito, 2007). This might lead to greater sensitivity of simple materials to manipulations that disrupt interitem processing (in ways posited by the item-order and multifactor accounts). These speculations aside, our central result is that the effects of generation and perceptual interference were uninfluenced by varying the retrieval context.

\section{AUTHOR NOTE}

Address correspondence to N. W. Mulligan, Department of Psychology, University of North Carolina, Chapel Hill, NC 27599-3270 (e-mail: nmulligan@unc.edu).

\section{REFERENCES}

Battig, W. F., \& Montague, W. E. (1969). Category norms for verbal items in 56 categories: A replication and extension of the Connecticut 
category norms. Journal of Experimental Psychology Monographs, 80(3, Pt. 2), 1-46.

BEgG, I., \& SNIDER, A. (1987). The generation effect: Evidence for generalized inhibition. Journal of Experimental Psychology: Learning, Memory, \& Cognition, 13, 553-563.

BegG, I., Snider, A., Foley, F., \& Goddard, R. (1989). The generation effect is no artifact: Generating makes words distinctive. Journal of Experimental Psychology: Learning, Memory, \& Cognition, 15, 977-989.

Bertsch, S., Pesta, B. J., Wiscott, R., \& McDaniel, M. A. (2007). The generation effect: A meta-analytic review. Memory \& Cognition, 35, 201-210.

Burns, D. J. (1990). The generation effect: A test between single- and multifactor theories. Journal of Experimental Psychology: Learning, Memory, \& Cognition, 16, 1060-1067.

Cox, S. D., \& Wollen, K. A. (1981). Bizarreness and recall. Bulletin of the Psychonomic Society, 18, 244-245.

DeLosh, E. L., \& McDaniel, M. A. (1996). The role of order information in free recall: Application to the word-frequency effect. Journal of Experimental Psychology: Learning, Memory, \& Cognition, 22, 1136-1146.

deWinstanley, P. A., BJork, E. L., \& Bjork, R. A. (1996). Generation effects and the lack thereof: The role of transfer-appropriate processing. Memory, 4, 31-48.

ENGelKamp, J., \& Dehn, D. M. (2000). Item and order information in subject-performed tasks and experimenter-performed tasks. Journal of Experimental Psychology: Learning, Memory, \& Cognition, 26, 671-682.

EngelKamp, J., Jahn, P., \& Seiler, K. H. (2003). The item-order hypothesis reconsidered: The role of order information in free recall. Psychological Research, 67, 280-290.

ERLEBACHER, A. (1977). Design and analysis of experiments contrasting the within- and between-subjects manipulation of the independent variable. Psychological Bulletin, 84, 212-219.

Gardiner, J. M., \& Hampton, J. A. (1988). Item-specific processing and the generation effect: Support for a distinctiveness account. American Journal of Psychology, 101, 495-504.

Hadley, C. B., \& MacKay, D. G. (2006). Does emotion help or hinder immediate memory? Arousal versus priority-binding mechanisms. Journal of Experimental Psychology: Learning, Memory, \& Cognition, 32, 79-88.

Healy, A. F., Shea, K. M., Kole, J. A., \& Cunningham, T. F. (2008). Position distinctiveness, item familiarity, and presentation frequency affect reconstruction of order in immediate episodic memory. Journal of Memory \& Language, 58, 746-764.

Hirshman, E., \& BJORK, R. A. (1988). The generation effect: Support for a two-factor theory. Journal of Experimental Psychology: Learning, Memory, \& Cognition, 14, 484-494.

Hirshman, E., \& Mulligan, N. W. (1991). Perceptual interference improves explicit memory but does not enhance data-driven processing. Journal of Experimental Psychology: Learning, Memory, \& Cognition, 17, 507-513.

Hirshman, E., Trembath, D., \& Mulligan, N. W. (1994). Theoretical implications of the mnemonic benefits of perceptual interference. Journal of Experimental Psychology: Learning, Memory, \& Cognition, 20, 608-620.

Hunt, R. R., \& Elliott, J. M. (1980). The role of nonsemantic information in memory: Orthographic distinctiveness effects on retention. Journal of Experimental Psychology: General, 109, 49-74.

Hunt, R. R., \& McDaniel, M. A. (1993). The enigma of organization and distinctiveness. Journal of Memory \& Language, 32, 421-445.

Kinoshita, S. (1989). Generation enhances semantic processing? The role of distinctiveness in the generation effect. Memory \& Cognition, 17, 563-571

KUČERA, H., \& FRANCIS, W. N. (1967). Computational analysis of presentday American English. Providence, RI: Brown University Press.

MCDANIEL, M. A., \& BugG, J. M. (2008). Instability in memory phenomena: A common puzzle and a unifying explanation. Psychonomic Bulletin \& Review, 15, 237-255.

McDaniel, M. A., DeLosh, E. L., \& Merritt, P. S. (2000). Order information and retrieval distinctiveness: Recall of common versus bizarre material. Journal of Experimental Psychology: Learning, Memory, \& Cognition, 26, 1045-1056.
McDaniel, M. A., Dornburg, C. C., \& GuYnn, M. J. (2005). Disentangling encoding versus retrieval explanations of the bizarreness effect: Implications for distinctiveness. Memory \& Cognition, 33, 270-279.

McDaniel, M. A., \& Einstein, G. O. (1986). Bizarre imagery as an effective memory aid: The importance of distinctiveness. Journal of Experimental Psychology: Learning, Memory, \& Cognition, 12, $54-65$.

McDaniel, M. A., \& Geraci, L. (2006). Encoding and retrieval processes in distinctiveness effects: Toward an integrative framework. In R. R. Hunt \& J. B. Worthen (Eds.), Distinctiveness and memory. (pp. 65-88). New York: Oxford University Press.

MCDANiel, M. A., Riegler, G. L., \& WADDILL, P. J. (1990). Generation effects in free recall: Further support for a three-factor theory. Journal of Experimental Psychology: Learning, Memory, \& Cognition, 16, 789-798.

Mulligan, N. W. (1996). The effects of perceptual interference at encoding on implicit memory, explicit memory, and memory for source. Journal of Experimental Psychology: Learning, Memory, \& Cognition, 22, 1067-1087.

Mulligan, N. W. (1999). The effects of perceptual interference at encoding on organization and order: Investigating the roles of itemspecific and relational information. Journal of Experimental Psychology: Learning, Memory, \& Cognition, 25, 54-69.

Mulligan, N. W. (2000). Perceptual interference and memory for order. Journal of Memory \& Language, 43, 680-697.

Mulligan, N. W. (2001). Generation and hypermnesia. Journal of Experimental Psychology: Learning, Memory, \& Cognition, 27, 436-450.

Mulligan, N. W. (2002a). The emergence of item-specific encoding effects in between-subjects designs: Perceptual interference and multiple recall tests. Psychonomic Bulletin \& Review, 9, 375-382.

Mulligan, N. W. (2002b). The emergent generation effect and hypermnesia: Influences of semantic and nonsemantic generation tasks. Journal of Experimental Psychology: Learning, Memory, \& Cognition, 28, 541-554.

Mulligan, N. W., \& Lozito, J. P. (2004). Self-generation and memory. In B. H. Ross (Ed.), The psychology of learning and motivation: Advances in research and theory (Vol 45, pp. 175-214). San Diego: Academic Press.

Mulligan, N. W., \& Lozito, J. P. (2007). Order information and free recall: Evaluating the item-order hypothesis. Quarterly Journal of Experimental Psychology, 60, 732-751.

NAIRNE, J. S. (1988). The mnemonic value of perceptual identification. Journal of Experimental Psychology: Learning, Memory, \& Cognition, 14, 248-255.

Nairne, J. S., Riegler, G. L., \& Serra, M. (1991). Dissociative effects of generation on item and order retention. Journal of Experimental Psychology: Learning, Memory, \& Cognition, 17, 702-709.

NAIRNE, J. S., \& WidNer, R. L. (1988). Familiarity and lexicality as determinants of the generation effect. Journal of Experimental Psychology: Learning, Memory, \& Cognition, 14, 694-699.

PeynirCioğLu, Z. F., \& Mungan, E. (1993). Familiarity, relative distinctiveness, and the generation effect. Memory \& Cognition, 21, 367-374.

RoEDIGER, H. L., III (2008). Relativity of remembering: Why the laws of memory vanished. Annual Review of Psychology, 59, 225-254.

Roenker, D. L., Thompson, C. P., \& Brown, S. C. (1971). Comparison of measures for the estimation of clustering in free recall. Psychological Bulletin, 76, 45-48.

SCHMidT, S. R. (1994). Effects of humor on sentence memory. Journal of Experimental Psychology: Learning, Memory, \& Cognition, 20, 953-967.

SCHMidT S. R. (in press). Distinctiveness and memory: A theoretical and empirical review. In J. H. Byrne (Series Ed.) \& H. L. Roediger III (Vol. Ed.), Learning and memory: A comprehensive reference. Vol. 2: Cognitive psychology of memory. San Diego: Academic Press.

Slamecka, N. J., \& Graf, P. (1978). The generation effect: Delineation of a phenomenon. Journal of Experimental Psychology: Human Learning \& Memory, 4, 592-604.

SlamecKa, N. J., \& Katsaiti, L. T. (1987). The generation effect as an artifact of selective displaced rehearsal. Journal of Memory \& Language, 26, 589-607.

Steffens, M. C., \& ERdfelder, E. (1998). Determinants of positive 
and negative generation effects in free recall. Quarterly Journal of Experimental Psychology, 51A, 705-733.

Talmi, D., LuK, B. T. C., McGarry, L. M., \& Moscovitch, M. (2007). The contribution of relatedness and distinctiveness to emotionallyenhanced memory. Journal of Memory \& Language, 56, 555-574.

Westerman, D. L., \& Greene, R. L. (1997). The effects of visual masking on recognition: Similarities to the generation effect. Journal of Memory \& Language, 37, 584-596.

WORTHEN, J. B. (2006). Resolution of discrepant memory strengths: An explanation of the effects of bizarreness on memory. In R. R. Hunt \& J. B. Worthen (Eds.), Distinctiveness and memory (pp. 133-156). New York: Oxford University Press.

\section{NOTES}

1. A full discussion of these accounts is, of course, beyond our present scope. However, it should be noted that both accounts adduce support from several lines of research independent of design effects (see Hunt \& McDaniel, 1993; McDaniel \& Bugg, 2008; Mulligan \& Lozito, 2004, for reviews).

2. That is, one is not asked to "remember what happened." Such a retrieval cue is too unconstrained to elicit useful information. Rather, one is asked to remember what happened at a particular time, at a particular place, or during a particular event. Such constraints establish the retrieval set (see Hunt \& McDaniel, 1993, for a discussion).
3. The critical, old words were counterbalanced across encoding conditions (as in Experiment 1), but not across old-new status. The latter is not a problem for the present experiment, because we are not concerned with absolute levels of recognition performance. We are interested only in the relative levels of recognition across the two encoding conditions.

4. An analysis using the lenient scores in the separate-recall condition produced the same results with one exception: The effect of test condition was also significant $[F(1,30)=5.91]$, indicating higher overall recall in the separate than in the combined test condition. This difference was not found for stringent or lenient scoring in Experiments 1, 4, or 5 . Consequently, we consider the difference found in this one experiment unimportant. Most importantly, the lenient scores, like the stringent scores, failed to produce an encoding-retrieval interaction $(F<1)$.

5. It should be noted that in the separate condition, no list confusion errors were reported. This is consistent with the notion that the lists were differentiated more easily in Experiment 4 than in Experiment 3. Of course, list confusion errors could also be averted postretrieval via output monitoring (i.e., a strategy of withholding retrieved items from the wrong category), so this datum is not definitive.

(Manuscript received February 29, 2008; revision accepted for publication July 7, 2008.) 